\title{
One-Stop Management with Perfusion for Transfer Patients with Stroke due to a Large-Vessel Occlusion: Feasibility and Effects on In-Hospital Times
}

\author{
(D) A. Brehm, (DI. Tsogkas, (DI.L. Maier, (D) H.J. Eisenberger, (DP. Yang, (D) J.-M. Liu, (D). Liman, and (D) M.-N. Psychogios
}

\begin{abstract}
BACKGROUND AND PURPOSE: In-hospital time delays lead to a relevant deterioration of neurologic outcomes in patients with stroke with large-vessel occlusions. At the moment, CT perfusion is relevant in the triage of late-window patients with stroke. We conducted this study to determine whether one-stop management with perfusion is feasible and leads to a reduction of in-hospital times.
\end{abstract}

MATERIALS AND METHODS: In this observational study, we report the first 15 consecutive transfer patients with stroke with externally confirmed large-vessel occlusions who underwent flat panel detector CT perfusion and thrombectomy in the same room. Preinterventional imaging consisted of noncontrast flat panel detector CT and flat panel detector CT perfusion, acquired with a biplane angiography system. The flat panel detector CT perfusion was used to reconstruct a flat panel detector CT angiography to confirm the large-vessel occlusions. After confirmation of the large-vessel occlusion, the patient underwent mechanical thrombectomy. We recorded time metrics and safety parameters prospectively and compared them with those of transfer patients whom we treated before the introduction of one-stop management with perfusion.

RESULTS: Fifteen transfer patients underwent flat panel detector CT perfusion and were treated with mechanical thrombectomy from June 2017 to January 2019. The median time from symptom onset to admission was 241 minutes. Median door-to-groin time was 24 minutes. Compared with 23 transfer patients imaged with multidetector CT, it was reduced significantly ( 24 minutes; $95 \%$ Cl, $19-37$ minutes, versus 53 minutes; $95 \% \mathrm{Cl}, 44-66$ minutes; $P<.001)$. Safety parameters were comparable between groups.

CONCLUSIONS: In this small series, one-stop management with perfusion led to a significant reduction of in-hospital times compared with our previous workflow.

ABBREVIATIONS: $\mathrm{FPDCT}=$ flat panel detector $\mathrm{CT} ; \mathrm{ICH}=$ intracranial hemorrhage; $\mathrm{IQR}=$ interquartile range; $\mathrm{LVO}=$ large-vessel occlusion; $\mathrm{MDCT}=$ multidetector $\mathrm{CT}$

A fter the publication of 5 landmark trials in 2015, thrombectomy became the criterion standard for the treatment of acute ischemic stroke due to a large-vessel occlusion (LVO) in the first 6 hours. ${ }^{1}$ Although the treatment effect has been shown to be time-dependent, ${ }^{2}$ door-to-groin times in the most recent studies remained $>100$ minutes. ${ }^{3}$ Therefore, we previously described a one-stop management ${ }^{4}$ for patients with acute stroke, which can

Received April 30, 2019; accepted after revision June 13.

From the Departments of Neuroradiology (A.B., I.T., H.J.E., M.-N.P.) and Neurology (I.L.M., H.J.E., J.L.), University Medical Center Goettingen, Goettingen, Germany; Department of Neurosurgery (P.Y., J.-M.L.), Changhai Hospital, Second Military Medical University, Changhai, China; and Department of Neuroradiology (A.B., I.T., M.-N.P.), Clinic of Radiology and Nuclear Medicine, University Hospital Basel, Basel, Switzerland.

Please address correspondence to Alex Brehm, MD, Department of Neuroradiology, University Medical Center Goettingen, Robert-Koch-Str 40, 37075, Goettingen, Germany; e-mail: Alex.brehm@med.uni-goettingen.de

- Indicates open access to non-subscribers at www.ajnr.org

7 Indicates article with supplemental on-line photo.

http://dx.doi.org/10.3174/ajnr.A6129 reduce door-to-groin times to $20-30$ minutes by combining diagnostic work-up and endovascular treatment in 1 room. ${ }^{5}$ One drawback of the previously described setup, especially in patients with a late window, ${ }^{6}$ was that CT perfusion imaging was not available on flat panel detector CT (FPDCT). This made one-stop management for patients in the extended time window, who were often transfer patients, impractical. In this observational study, we report the time metrics and safety parameters of the first 15 consecutive transfer patients who were transported directly to the angiosuite and underwent FPDCT perfusion before their endovascular treatment as part of their diagnostic work-up.

\section{MATERIALS AND METHODS \\ Patient Selection}

This retrospective observational study includes all consecutive adults who were transfer patients and underwent FPDCT perfusion from June 2017 to January 2019. All patients underwent multidetector CT with angiography at the referring center before arrival at our center. In European stroke centers, it is common to 
repeat imaging (including CT perfusion) before thrombectomy in patients with an interhospital delay of $>60$ minutes, to ensure that they still meet the eligibility criteria. As part of our protocol after the introduction of FPDCT perfusion, transfer patients with an interhospital delay of $>60$ minutes and with a NIHSS score of $>7$ were triaged directly to the angiosuite. Data were extracted from a prospectively acquired institutional review board-approved data base. As a control group, we used transfer patients treated endovascularly between January 2016 and June 2017, before the introduction of FPDCT perfusion, presenting with an NIHSS score of $>7$ whom we triaged with multidetector CT (MDCT). In both groups, occupation of the angiosuite during arrival of the patients was an exclusion criterion. One experienced neuroradiologist (I.T. with $>5$ years of experience) rated FPDCT perfusion and FPDCT angiography regarding overall diagnostic quality, diagnostic quality of the FPDCT perfusion, motion artifacts, LVO detection, and evaluation of collaterals on a 5 -point ordinal scale $(0=$ no diagnostic quality, $1=$ poor diagnostic quality, 2 = fair diagnostic quality, $3=$ good diagnostic quality, 4 = perfect diagnostic quality). The patient's consent for treatment was obtained according to institutional guidelines. The local ethics committee waived the need for a formal application or a separate consent concerning the inclusion in our observational data base.

\section{Image Acquisition and Processing}

All FPDCT/FPDCT perfusion images were acquired using an Artis Q angiography system (Siemens, Erlangen, Germany). First, we performed a noncontrast FPDCT to rule out hemorrhage. A commercially available 20 -second rotational acquisition was used (20-second Dyna CT Head, $109 \mathrm{kV}, 1.8 \mu \mathrm{Gy} /$ frame, 200 ${ }^{\circ}$ angle, $0.4^{\circ}$ /frame angulation step, effective dose $\sim 2.5 \mathrm{mSv}$ ). The raw data were automatically and instantly reconstructed in 5-mm multiplanar reconstructions on a commercially available workstation (syngo X Workplace). Second, we performed the FPDCT perfusion (multisweep FPDCT perfusion with $10 \times 5$-second rotations, $77 \mathrm{kV}, 0.36 \mu \mathrm{Gy} /$ frame, $200^{\circ}$ angle, $0.5^{\circ} /$ frame angulation step, effective dose $\sim 5 \mathrm{mSv}$ ). The FPDCT perfusion protocol has been described in detail elsewhere. ${ }^{7,8}$

\section{Statistical Analysis}

We used descriptive statistics to illustrate patient characteristics and time metrics. In case of time intervals, we opted for median and interquartile ranges (IQRs) to describe them in detail. For the comparison of continuous study parameters, the $t$ test was used in the case of normal distribution, and the Mann-Whitney test, in the case of a non-normal or ordinal distribution. Categorical variables were compared with the 2 groups by Fischer exact test. The threshold for statistical significance was set at $P<.05$. We performed all analyses with MedCalc for Windows, Version 18, 2018 (MedCalc Software, Mariakerke, Belgium).

\section{RESULTS}

We included all 15 (mean age, 78 years; 7 women) transfer patients who underwent FPDCT perfusion after June 2017 in our study. The median NIHSS score at admission was 15 , and the native ASPECTS was 8 . Of the 15 patients, 7 arrived at our hospital within off-duty hours. The median symptom-to-door time was
Table 1: Baseline characteristics and time metrics of the patients with one-stop and MDCT perfusion ${ }^{\mathrm{a}}$

\begin{tabular}{|c|c|c|c|}
\hline & $\begin{array}{c}\text { One-Stop } \\
\text { Perfusion } \\
(n=15)\end{array}$ & $\begin{array}{c}\text { MDCT } \\
\text { Perfusion } \\
(n=23)\end{array}$ & $\begin{array}{c}P \\
\text { Value }\end{array}$ \\
\hline \multicolumn{4}{|l|}{ Baseline characteristics } \\
\hline Female (No.) & 7 & 9 & 1 \\
\hline Age (yr) & $78(68-88)$ & $68(61-78)$ & $.048^{\mathrm{b}}$ \\
\hline NIHSS_a & 15 (12-19) & $18(14-24)$ & .117 \\
\hline CCT-Ā̄PECTS & $8(8-9)$ & $7(6-9)$ & .149 \\
\hline Symptom to door & $241(204-282)$ & $248(175-327)$ & .784 \\
\hline Other CT to in-house $\mathrm{CT}$ & 133 (114-156) & 170 (145-327) & .06 \\
\hline \multicolumn{4}{|l|}{ Time metrics } \\
\hline Door to $\mathrm{CT}$ & $11(8-25)$ & $15(11-25)$ & .129 \\
\hline Door to groin & $24(19-34)$ & $53(41-69)$ & $<.001^{\mathrm{b}}$ \\
\hline In-house $\mathrm{CT}$ to groin & $14(10-22)$ & $34(22-43)$ & $<.001^{\mathrm{b}}$ \\
\hline Door to reperfusion & $76(59-95)$ & $97(81-122)$ & .078 \\
\hline Groin to reperfusion & $41(33-76)$ & $54(29-68)$ & .578 \\
\hline In-house CT to reperfusion & $55(47-85)$ & $80(63-106)$ & .083 \\
\hline \multicolumn{4}{|l|}{ Safety parameters } \\
\hline $\mathrm{sICH}$ & $1(6.7 \%)$ & $2(8.7 \%)$ & 1 \\
\hline Mortality & $4(26.7 \%)$ & $4(17.4 \%)$ & .114 \\
\hline SAH on follow-up scan & $1(6.7 \%)$ & $3(13 \%)$ & 1 \\
\hline
\end{tabular}

Note:-CCT indicates cranial CT; NIHSS_a, admission NIHSS; Other CT to in-house $\mathrm{CT}$, external $\mathrm{CT}$ to our $\mathrm{CT}$; $\mathrm{sICH}$, symptomatic intracranial hemorrhage.

${ }^{\text {a }}$ Data are median and IQR or No. (\%).

${ }^{\mathrm{b}}$ Significant.

241 minutes (IQR, 204-282 minutes), while the median time from the external CT to our FPDCT was 133 minutes (IQR, 114156 minutes) (Table 1). The median time between the registration in the hospital information system and the reconstruction of the first FPDCT slices was 11 minutes (IQR, 8-25 minutes). In all 15 cases, we confirmed the external diagnosis of an LVO. In 1 case, we detected an intracranial hemorrhage $(\mathrm{ICH})$, which was not present on the external CT scan (On-line Figure). However, all 15 cases proceeded to endovascular treatment because the ICH was on the hemisphere contralateral to the LVO. We achieved successful reperfusion (defined as a modified TICI $\geq 2 \mathrm{~b}$ ) in 11 of 15 cases, with median groin-to-reperfusion and door-to-reperfusion times of 41 minutes (IQR, 33-76 minutes) and 76 minutes (IQR, 59-95 minutes), respectively. Five of the reperfused patients presented with a tandem occlusion. We observed 1 symptomatic ICH and $1 \mathrm{SAH}$ in this group. The mortality rate was 4 of 15 (26.7\%).

The control group consisted of 23 transfer patients (mean age, 68 years; 9 women), with a median symptom-to-door time of 248 minutes (IQR, 175-327 minutes) and a median time from the external CT to our MDCT of 170 minutes (IQR, 145-327 minutes). All patients in the control group had an externally confirmed LVO. The median time between registration in the hospital information system and reconstruction of the first MDCT slide was 15 minutes (IQR, 11-25 minutes; $P=.129$ ) and therefore comparable. All other baseline parameters, excluding age, which was significantly higher in the one-stop management group (78 years; IQR, $68-88$ years, versus 68 years; IQR, $61-78$ years; $P=$ $.048)$, were comparable between groups.

In the one-stop management with perfusion group, $80 \%$ were treated with the Stent retriever Assisted Vacuum-locked Extraction ${ }^{9}$ (SAVE) technique (a primary combined approach of a stent retriever and an aspiration catheter), while the remaining 20\% were treated with A Direct Aspiration first Pass Technique (ADAPT) as a first-pass strategy. In the control group, $65.2 \%$ were treated with SAVE, while 
Table 2: Subjective evaluation of the quality of the FPDCT angiography and perfusion

\begin{tabular}{lccccc}
\multicolumn{1}{c}{ Variable } & $\begin{array}{c}\text { No Diagnostic } \\
\text { Quality/Severe }\end{array}$ & $\begin{array}{c}\text { Poor Diagnostic } \\
\text { Quality/Substantial }\end{array}$ & $\begin{array}{c}\text { Fair Diagnostic } \\
\text { Quality/Tolerable }\end{array}$ & $\begin{array}{c}\text { Good Diagnostic } \\
\text { Quality/Negligible }\end{array}$ & $\begin{array}{c}\text { Perfect Diagnostic } \\
\text { Quality/None }\end{array}$ \\
\hline FPDCT angiography/perfusion overall & 0 & 1 & 3 & 3 & 7 \\
Detection of LVO & 0 & 0 & 3 & 5 & 4 \\
Evaluation of collaterals & 0 & 0 & 3 & 6 & 6 \\
FPDCT perfusion & 1 & 1 & 4 & 5 & 4 \\
Motion artifacts & 1 & 2 & & 3 \\
\hline
\end{tabular}

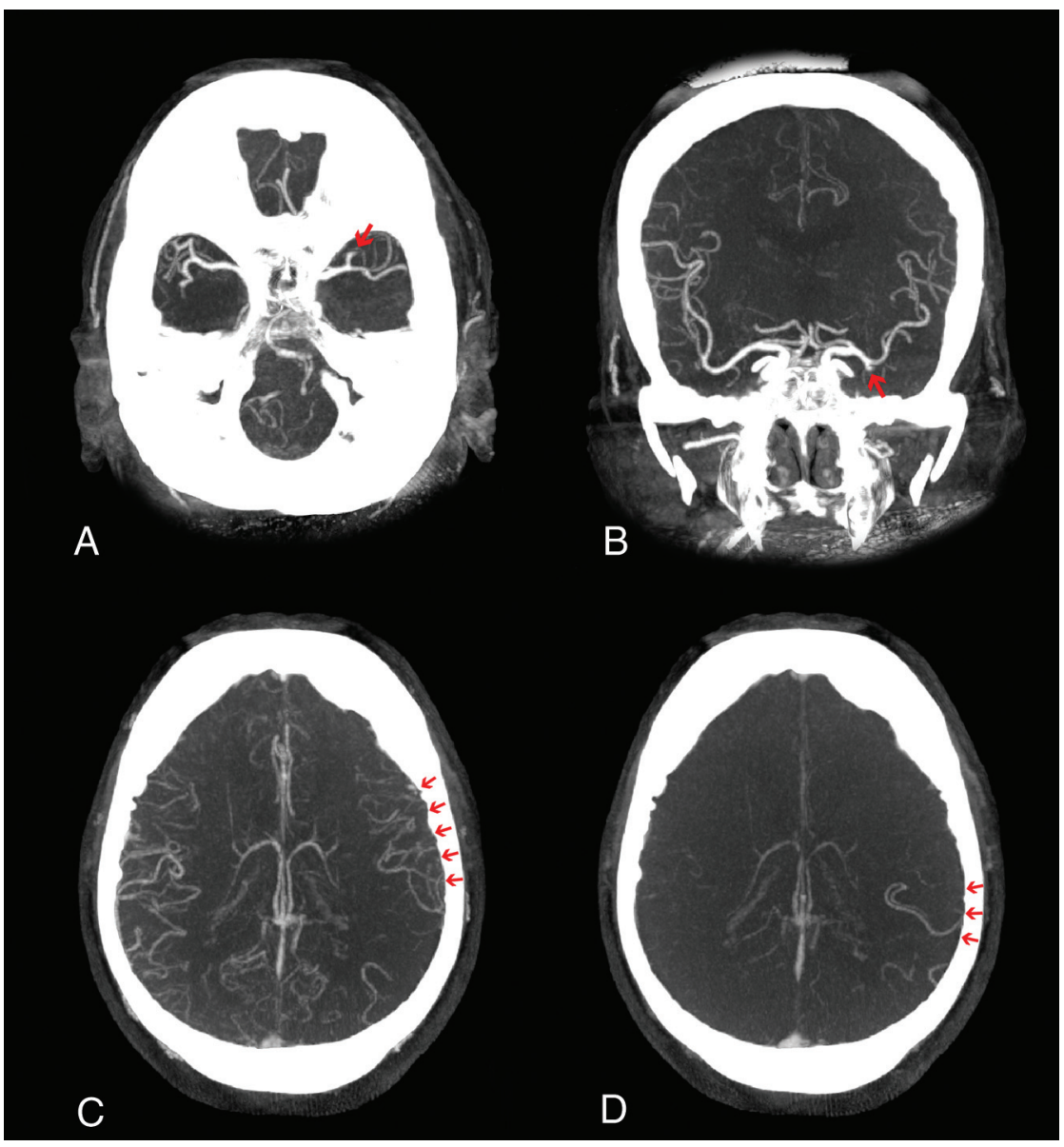

FIG 1. FPDCT angiography for the identification of an LVO on a transversal FPDCT angiogram (A), a coronal FPDCT angiogram $(B)$, and early-phase $(C)$ and late-phase $(D)$ collaterals. and mortality $(26.7 \%$ versus $17.4 \% ; P=$ $.114)$ did not differ significantly between groups.

The diagnostic quality was sufficient for the delineation of the LVO and the evaluation of collaterals in all cases (Table 2$)$. In 11 of 15 cases $(73.3 \%)$, the overall diagnostic quality was at least good, while it was fair in 3 cases $(20 \%)$ and poor in 1 case $(7.7 \%)$, due to heavy motion artifacts. One of 15 FPDCT perfusions was not of diagnostic quality due to heavy motion artifacts.

\section{DISCUSSION}

In this small, observational study, onestop management with perfusion for transfer patients with a confirmed LVO was safe and significantly shortened door-to-groin times compared with transfer patients imaged with an MDCT perfusion protocol. The reported door-to-groin times are comparable to the door-to-groin times, which we achieved with our original one-stop management with a biphasic FPDCT angiography protocol (20.5 minutes; 95\% CI, 17-26 minutes). ${ }^{5}$ Acquisition and reconstruction of the FPDCT perfusion did not result in a relevant time loss. Besides, they are well below those times in recent randomized controlled trials the remaining 34.8\% were treated with A Direct Aspiration first Pass Technique (ADAPT) as a first-pass strategy. There was no statistically significant difference between groups $(P=.333)$. Of the control group, $52.2 \%$ received general anesthesia, while $46.7 \%$ of the onestop management with perfusion group received general anesthesia $(P=1.0)$.

We were able to significantly reduce median door-to-groin times from 53 minutes (IQR, 41-69 minutes) in the MDCT group to 24 minutes (IQR, 19-34 minutes) in the one-stop management group $(P<.001)$. In-house median CT-to-groin times were significantly reduced as well from 34 minutes (IQR, 22-43 minutes) to 14 minutes (IQR, $10-22$ minutes) $(P<.001)$. The impact on door-to-reperfusion times was also relevant; however, it was not statistically significant (76 minutes; IQR, 59-95 minutes, versus 97 minutes; IQR, 81-122 minutes; $P=.078)$.

The frequencies of symptomatic ICH $(6.7 \%$ versus $8.7 \% ; P=$ 1), SAH observed on a follow-up scan (6.7\% versus $13 \%$; $P=1)$, with median door-to-groin times of $>60$ minutes, even for transfer patients. ${ }^{2,3,10}$ The reduction of door-to-reperfusion times was relevant, but not statistically significant $(P=.078)$. However, this has to be attributed to the small sample size and the high prevalence of carotid-terminus and tandem occlusion in this small sample. This argument is strengthened because imaging-to-reperfusion times were shorter as well with 55 minutes compared with 80 minutes $(P=.083)$, which is well below the $<90$ minutes suggested by Goyal et al. ${ }^{11}$ According to Guenego et al, ${ }^{12}$ the main subgroup of transfer patients in which repeat perfusion imaging is necessary are those with fast-progressing strokes because they might miss the eligible criteria after transfer to the comprehensive stroke center. Saving 30 minutes of in-hospital time in this cohort of patients might result in significantly better clinical outcomes, strengthening the argument for a direct transfer to the angiosuite for diagnostic work-up and mechanical thrombectomy. 


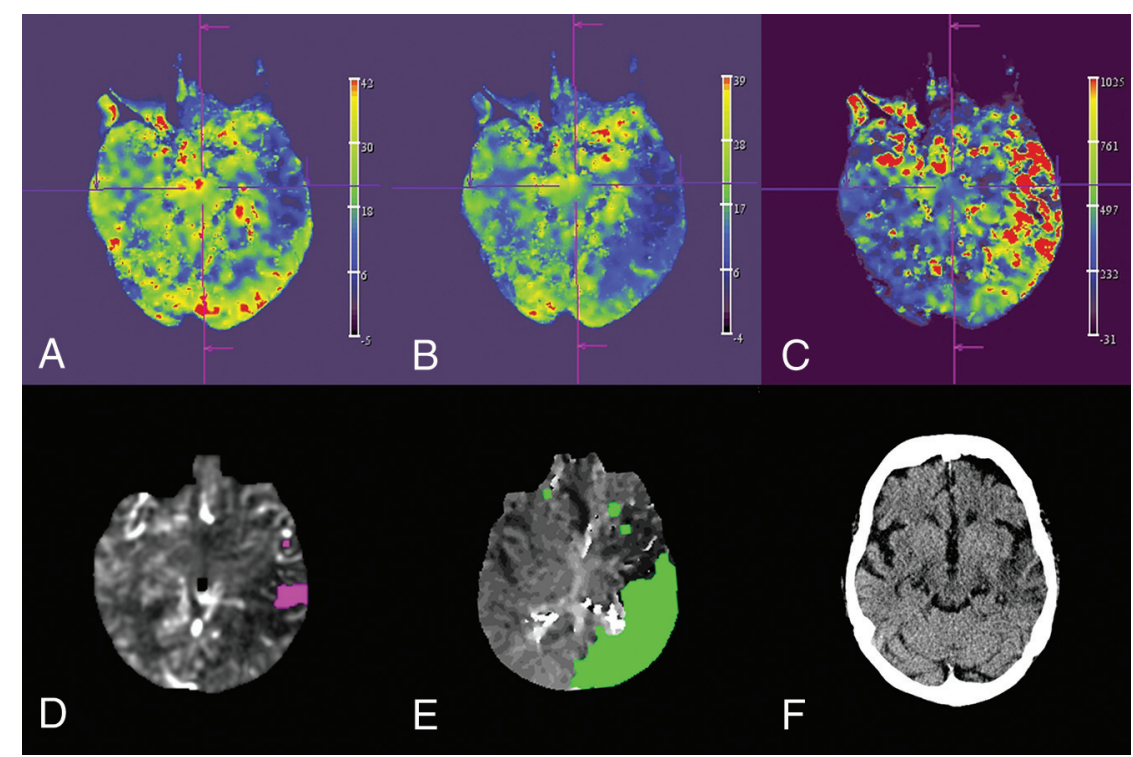

FIG 2. Reconstruction of the $C B V(A), C B F(B)$, and $M T T(C)$ map by the syngo $X$ Workplace (Siemens). Visualization of the $10-\mathrm{mL}$ infarct core $(D)$, the $225-\mathrm{mL}$ mismatch volume $(E)$ by the RAPID ANGIO software solution, and the final infarction on a follow-up CT scan 2 days after the thrombectomy $(F)$.

Regarding the differentiation between ischemic and hemorrhagic stroke, which is particularly important in "mothership" (direct admission) patients, previous work from our group showed that FPDCT detects ICH with a high sensitivity and specificity, comparable with MDCT. ${ }^{13}$ Furthermore the FPDCT perfusion dataset can be used to reconstruct FPDCT angiography as was pointed out by Yang et $\mathrm{al}^{14}$ in a prior publication. In our sample, the quality of the reconstructed FPDCT angiography was sufficient to delineate the LVO in all cases and evaluate collaterals (Fig 1 and Table 2). This confirms prior results of other work groups who used it successfully for evaluating collaterals and the clot-burden score. ${ }^{15,16}$ Collaterals can be used as an additional tool for patient selection as pointed out by Schregel et al, ${ }^{17}$ who compared the prognostic value of CBV-ASPECTS and collaterals. Although the time resolution of the FPDCT perfusion is 5 seconds lower than the time resolution of the MDCT Perfusion (1.5 seconds), motion artifacts were not a serious issue in our small cohort. Overall the diagnostic quality of the reconstructed FPDCT perfusion (Fig 2) was sufficient in 14 of 15 cases (93.3\%). Furthermore, the next generation of FPDCT is reported to have faster acquisition times, which might improve the time resolution of the FPDCT perfusion protocol. Regarding automated evaluation of FPDCT perfusion, the RAPID ANGIO software solution (iSchemaView, Inc) was presented recently (Fig 2 ) ${ }^{18}$ providing swift and automated calculation of infarct core and mismatch. The reliability and accuracy of RAPID ANGIO for the exact depiction of core and penumbra are the subject of running studies. The effective dosage of the FPDCT perfusion protocol is comparable with the effective dosage of an MDCT perfusion protocol. ${ }^{19,20}$

Another interesting aspect of our approach is that FPDCT angiography can be projected into the angiosuite, giving the treating physician important information about the vessel architecture, which can be particularly helpful in case of an occlusion of the terminus of the internal carotid artery because the down- stream vessel architecture cannot be visualized with DSA. Even in case of an M1 occlusion, information about the downstream vessel architecture can be helpful to navigate the stent retriever into the more prominent truncus, which might improve angiographic outcomes. ${ }^{21}$

The primary limitations of our study are the observational design, which is prone to selection bias, and the small number of reported patients.

"Time is brain" is the mantra that was propagated by Saver et $\mathrm{al}^{2}$, who showed a tight correlation between door-to-reperfusion times and outcome. Even in our setting with the MDCT scanner only 20 meters away from the angiosuite, we were able to significantly reduce door-to-groin times compared with traditionally triaged patients, despite the fact that we had optimized the MDCT route with standardization and weekly training before introduction of the one-stop management. ${ }^{22}$ Effects on door-to-groin times should be even more profound in other hospital settings, where the MDCT scanner is far away from the angiosuite or even located in another building. However, a prospective study is warranted to confirm the observed differences in a larger cohort and examine its effect on outcome. Furthermore, the equivalence of FPDCT and MDCT perfusion is still a topic of ongoing investigation.

\section{CONCLUSIONS}

One-stop management with perfusion for transfer patients with a confirmed LVO in a modern angiosuite is feasible and safe and leads to a significant reduction of door-to-groin times.

Disclosures: Alex Brehm—UNRELATED: Grant: research agreement with Siemens.* Hanna J. Eisenberger-OTHER RELATIONSHIPS: member of the Deutsche Gesellschaft für Neurologie. Jan Liman—UNRELATED: Expert Testimony: Pfizer; Payment for Lectures Including Service on Speakers Bureaus: Stryker, Bristol-Myers Squibb; Pfizer; Travel/Accommodations/Meeting Expenses Unrelated to Activities Listed: Bayer HealthCare Pharmaceuticals, Daiichi Sankyo. Marios-Nikos Psychogios-UNRELATED: Grant: Siemens, Comments: research grant with the Department of Neuroradiology*; Consulting Fee or Honorarium: Siemens. *Money paid to the institution.

\section{REFERENCES}

1. Goyal M, Menon BK, Van Zwam WH, et al; HERMES collaborators. Endovascular thrombectomy after large-vessel ischaemic stroke: a meta-analysis of individual patient data from five randomised trials. Lancet 2016;387:1723-31 CrossRef Medline

2. Saver JL, Goyal M, Van Der Lugt A, et al; HERMES collaborators. Time to treatment with endovascular thrombectomy and outcomes from ischemic stroke: a meta-analysis. JAMA 2016;316:1279-88 CrossRef Medline

3. Nogueira RG, Frei D, Kirmani JF, et al; Penumbra Separator 3D Investigators. Safety and efficacy of a 3-dimensional stent retriever with aspiration-based thrombectomy vs aspiration-based thrombectomy alone in acute ischemic stroke intervention: a randomized clinical trial. JAMA Neurol 2018;75:304-311 CrossRef Medline

4. Psychogios MN, Bähr M, Liman J, et al. One stop management in acute 
stroke: first mothership patient transported directly to the angiography suite. Clin Neuroradiol 2017;27:389-91 CrossRef Medline

5. Psychogios MN, Behme D, Schregel K, et al. One-stop management of acute stroke patients: minimizing door-to-reperfusion times. Stroke 2017;48:3152-55 CrossRef Medline

6. Nogueira RG, Jadhav AP, Haussen DC, et al; DAWN Trial Investigators. Thrombectomy $\mathbf{6}$ to 24 hours after stroke with a mismatch between deficit and infarct. $N$ Engl J Med 2017;378:11-21 CrossRef Medline

7. Struffert T, Deuerling-Zheng Y, Kloska S, et al. Dynamic angiography and perfusion imaging using flat detector $\mathrm{CT}$ in the angiography suite: a pilot study in patients with acute middle cerebral artery occlusions. AJNR Am J Neuroradiol 2015;36:1964-70 CrossRef Medline

8. Garcia M, Okell TW, Gloor M, et al. Feasibility of flat panel detector CT in perfusion assessment of brain arteriovenous malformations: initial clinical experience. AJNR Am J Neuroradiol 2017;38:735-39 CrossRef Medline

9. Maus V, Behme D, Kabbasch C, et al. Maximizing first-pass complete reperfusion with SAVE. Clin Neuroradiol 2018;28:327-38 CrossRef Medline

10. Lapergue B, Blanc R, Gory B, et al; ASTER Trial Investigators. Effect of endovascular contact aspiration vs stent retriever on revascularization in patients with acute ischemic stroke and large vessel occlusion: the ASTER randomized clinical trial. JAMA 2017;318: 443-52 CrossRef Medline

11. Goyal M, Menon BK, Hill MD, et al. Consistently achieving computed tomography to endovascular recanalization $<90$ minutes: solutions and innovations. Stroke 2014;45:e252-56 CrossRef Medline

12. Guenego A, Mlynash M, Christensen S, et al. Hypoperfusion ratio predicts infarct growth during transfer for thrombectomy. Ann Neurol 2018;84:616-20 CrossRef Medline

13. Leyhe JR, Tsogkas I, Hesse AC, et al. Latest generation of flat detector $\mathrm{CT}$ as a peri-interventional diagnostic tool: a comparative study with multidetector CT. J Neurointerv Surg 2017;9:1253-57 CrossRef Medline

14. Yang P, Niu K, Wu Y, et al. Time-resolved C-arm computed tomo- graphic angiography derived from computed tomographic perfusion acquisition: new capability for one-stop-shop acute ischemic stroke treatment in the angiosuite. Stroke 2015;46:3383-89 CrossRef Medline

15. Hoelter P, Goelitz P, Lang S, et al. Visualization of large vessel occlusion, clot extent, and collateral supply using volume perfusion flat detector computed tomography in acute stroke patients. Acta Radiol 2019 Mar 12:284185119836220. [Epub ahead of print] CrossRef Medline

16. Yang $\mathrm{P}, \mathrm{Niu} \mathrm{K}, \mathrm{Wu} \mathrm{Y}$, et al. Evaluation of collaterals and clot burden using time-resolved C-arm conebeam CT angiography in the angiography suite: a feasibility study. AJNR Am J Neuroradiol 2017;38: 747-52 CrossRef Medline

17. Schregel K, Tsogkas I, Peter C, et al. Outcome prediction using perfusion parameters and collateral scores of multi-phase and singlephase CT angiography in acute stroke: need for one, two, three, or thirty scans? J Stroke 2018;20:362-72 CrossRef Medline

18. Evers P. iSchemaView Launches RAPID ${ }^{\mathrm{TM}}$ ANGIO. BusinessWire. https://www.businesswire.com/news/home/20190228005091/en/ iSchemaView-Launches-RAPID\%E2\%84\%A2-ANGIO. Accessed April 2, 2019

19. Psychogios M, Schramm P, Frölich AM, et al. Alberta Stroke Program Early CT Scale evaluation of multimodal computed tomography in predicting clinical outcomes of stroke patients treated with aspiration thrombectomy. Stroke 2013;44:2188-93 CrossRef Medline

20. Struffert T, Hauer M, Banckwitz R, et al. Effective dose to patient measurements in flat-detector and multislice computed tomography: a comparison of applications in neuroradiology. Eur Radiol 2014;24:1257-65 CrossRef Medline

21. Maus V, Brehm A, Tsogkas I, et al. Stent retriever placement in embolectomy: the choice of the post-bifurcational trunk influences the first-pass reperfusion result in M1 occlusions. J Neurointerv Surg 2019;11:237-40 CrossRef Medline

22. Schregel K, Behme D, Tsogkas I, et al. Optimized management of Endovascular Treatment for Acute Ischemic Stroke. J Vis Exp 2018; 131:56397 Medline 